\title{
Emotion simulation during language comprehension
}

\author{
David A. Havas And Arthur M. Glenberg \\ University of Wisconsin, Madison, Wisconsin \\ AND \\ MiKe RINCK \\ Dresden University of Technology, Dresden, Germany
}

\begin{abstract}
We report a novel finding on the relation of emotion and language. Covert manipulation of emotional facial posture interacts with sentence valence when measuring the amount of time to judge valence (Experiment 1) and sensibility (Experiment 2) of the sentence. In each case, an emotion-sentence compatibility effect is found: Judgment times are faster when facial posture and sentence valence match than when they mismatch. We interpret the finding using a simulation account; that is, emotional systems contribute to language comprehension much as they do in social interaction. Because the effect was not observed on a lexical decision task using emotion-laden words (Experiment 3), we suggest that the emotion simulation affects comprehension processes beyond initial lexical access.
\end{abstract}

Reading a passage from a favorite novel makes it clear that language evokes emotion. In addition, empirical studies have shown that emotions that are evoked by language can be powerful (Velten, 1968) and can have an impact on judgments (Johnson \& Tversky, 1983). Nonetheless, the interaction between emotion and language is not well understood. Oatley (1994) suggests that since Aristotle, one explanation is based on the notion of simulation. We briefly review recent theory, data, and methods investigating simulation in language comprehension and in social cognition before introducing a new method to study the relation between emotion and language comprehension. In this article, we present initial evidence consistent with (1) a simulation account, and (2) the idea that the effect of emotion on language comprehension is likely to result from processes other than initial lexical access.

By "simulation during language comprehension," we mean that a neural process ordinarily having a nonlinguistic function (e.g., action, perception, emotion) is also used during language comprehension. In addition, the simulation may be functional in that it plays a causal role in language comprehension. As we will review shortly, the case for functional simulation is strongest in the domains of action and perception. The evidence that we present for simulation using emotion is only initial; that is, the functional role has yet to be secured.

The case for simulation of action during language comprehension is very strong because of converging evidence from behavioral, physiological, and neuroscience techniques. Glenberg and Kaschak (2002; see also Zwaan \& Taylor, 2006) demonstrated a relation between action and language comprehension by using behavioral methods. Participants read sentences describing action away from the body (e.g., You handed Courtney the notebook) or toward the body (Courtney handed you the notebook) and judged whether the sentences were sensible. Participants responded "sensible" by reaching toward a button far from the body in one condition and toward the body in the other condition. Participants were faster to make "sensible" judgments when the action that was implied by the sentence (e.g., away from the body) was compatible with the action required to make the response. These data support the claim that language comprehension involves simulation grounded in neural systems for action. Using fMRI, Hauk, Johnsrude, and Pulvermüller (2004) demonstrated that the motor and premotor cortex are activated in a somatotopic fashion while silently reading action verbs, such as kick, pick, and lick. That is, in addition to activation in traditional language areas, understanding the word kick initiated activation in areas of the motor and premotor cortex that control the leg, whereas understanding the word pick initiated activation in areas that control the hand, and so on. Evidence consistent with the claim that the simulation is functional comes from the use of transcranial magnetic stimulation (TMS). Thus, Buccino et al. (2005) demonstrated that sentences about hand action modulated muscle activation in the hand when a TMS pulse was applied to the hand motor area.

The case for the use of perceptual systems in language comprehension is also strong. Kaschak et al. (2005) asked participants to judge the sensibility of sentences describing motion in a particular direction (e.g., The train is

D.A. Havas, dahavas@wisc.edu 
approaching) while watching a visual display depicting matching or mismatching motion. Kaschak et al. (2005) found that the depicted motion affected the time needed to comprehend sentences describing motion in the same direction. Furthermore, Tettamanti et al. (2005) used fMRI to demonstrate that listening to sentences describing visible action selectively activates area V5, which is known to function in the perception of visual motion.

The case for emotion simulation during language comprehension is weaker. There is, for example, literature on the emotional Stroop effect, but there is controversy over its interpretation (see, e.g., Larsen, Mercer, \& Balota, 2006). There are limited interactions between mood and lexical decisions (Innes-Ker \& Niedenthal, 2002), but it is not clear whether other language processes are affected.

In contrast, the case for emotion simulation in social cognition is growing. For instance, simulation has been proposed as a mechanism for empathy (Decety \& Grezes, 2006). Using fMRI, Carr, Iacoboni, Dubeau, Mazziotta, and Lenzi (2003) found that observation and imitation of emotional facial expressions activate the same neural areas of emotion, as well as motor areas associated with the mirror neuron system (Rizzolatti, Fogassi, \& Gallese, 2001). Motor system activity is consistent with the finding that observed emotional facial expressions automatically activate viewers' facial musculature (Dimberg, 1982; Dimberg, Thunberg, \& Elmehed, 2000). This link is bidirectional: Self-generated facial expressions of emotion facilitate perception of those expressions appearing in others (Niedenthal, Brauer, Halberstadt, \& Innes-Ker, 2001). And Levenson, Ekman, and Freisen (1990) demonstrated that changing facial posture generates emotion-specific autonomic activity. Facial posture may thus be part of a functionally integrated emotion simulation system for producing adaptive social actions (de Gelder, 2006).

The work reported in this article is intended to help develop the claim that emotion simulation occurs and has a functional role in the domain of language comprehension by using a behavioral task. To do so, we measured the time needed to comprehend sentences describing emotionally laden events when the participant was in a matching or mismatching emotional state. We manipulated emotion using a procedure developed by Strack, Martin, and Stepper (1988) that involves holding a pen in the mouth to produce either a smile (holding the pen using only the teeth) or a frown or pout (holding the pen using only the lips and not the teeth). In comparison with a control condition, participants who were forced to smile reported greater intensity of felt humor in response to cartoons, and participants who were forced to frown reported less felt humor (Strack et al., 1988). This procedure has been shown to reliably influence positive and negative emotional experiences in the absence of conscious mediation (Strack et al., 1988). If emotion simulation occurs during language comprehension, we would expect to see an interaction such that the processing of pleasant sentences is faster when the pen is held in the teeth (and participants are smiling) than when the pen is held in the lips (so that smiling is prevented), and vice versa for the time to process unpleasant sentences.

\section{EXPERIMENT 1}

\section{Method}

Ninety-six participants from the University of Wisconsin, Madison, were given course credit for participating in a sentence judgment task. Sentences were displayed individually, and we timed how long it took the participant to decide whether the sentence described a pleasant or an unpleasant event. The two within-subjects independent variables were sentence valence (positive or negative) and pen condition (pen-in-lips or pen-in-teeth). Ninety-six stimulus sentences (48 pleasant and 48 unpleasant) were based on those constructed and normed by Fischler. ${ }^{1}$

\author{
Example pleasant sentences: \\ The college president announces your name, and you proudly \\ step onto the stage. \\ You and your lover embrace after a long separation. \\ Example unpleasant sentences: \\ The police car rapidly pulls up behind you, siren blaring. \\ Your supervisor frowns as he hands you the sealed envelope.
}

In their norming procedure, approximately 60 participants rated each of the original sentences on a scale from 1 (most unpleasant) to 9 (most pleasant). The mean was $2.92(S D=.66)$ for the unpleasant sentences and $6.50(S D=.58)$ for the pleasant sentences.

Participants were instructed on how to hold a pen in the mouth using just the teeth and just the lips. They were told that the purpose of the pen manipulation was to investigate how the suppression of speech articulators affects sentence processing. Sentences were presented one at a time on a computer monitor. The participants initiated the presentation of a sentence by pressing the space bar, which also started a timer. The participants used the index fingers of both hands to judge the valence of each sentence as either "pleasant" or "unpleasant" by pressing the " 3 " key (marked "U") or the " 0 " key (marked "P") on the computer keyboard. Half of the participants began with the pen in their teeth, and half with the pen in their lips. After every block of twelve trials ( 6 pleasant and 6 unpleasant sentences, randomly intermixed), participants were instructed to switch pen condition. This instruction also enabled the experimenter to ensure participants' adherence to the pen manipulation.

\section{Results and Discussion}

On average, participants were $96 \%$ correct. Only correct trials were used in the analysis of judgment times, and other trials were eliminated when the judgment time was greater than $2.5 S D$ s from a participant's mean for that condition. The results of Experiment 1 (Table 1) demonstrated an effect of emotions on speed of the judgment. The critical interaction between the pen condition and sentence valence was significant $\left[F_{1}(1,95)=4.41, M S_{\mathrm{e}}=\right.$ $87,320, p=.03] .{ }^{2}$ Pleasant sentences were read $54 \mathrm{msec}$ faster when participants were smiling (pen-in-teeth) than when they were frowning (pen-in-lips); unpleasant sentences were read $36 \mathrm{msec}$ slower when participants were smiling than when they were frowning.

Table 1

Judgment Times (RTs, in Milliseconds) and Proportions of Consistent Valence Judgments (Judg.) From Experiment 1

\begin{tabular}{cccccc}
\hline & \multicolumn{3}{c}{ Sentence Valence } \\
\cline { 2 - 3 } \cline { 5 - 6 } Pen Condition & RT & Judg. & & RT & Judg. \\
\cline { 2 - 3 } \cline { 5 - 6 } & Rnpleasant & & & Und \\
Teeth (smiling) & 2,679 & .96 & & 2,684 & .97 \\
Lips (frowning) & 2,733 & .97 & & 2,648 & .97 \\
\hline
\end{tabular}


One could argue that the observed emotional congruency effect is not a product of comprehension per se, but of the task requirement to judge the emotional valence of the sentences. Therefore, in Experiment 2, the instructions made no reference to the emotional valence of the sentences.

\section{EXPERIMENT 2}

\section{Method}

Experiment 2 was identical to Experiment 1 except that the 42 participants were instructed to respond to the sentences as either "easy" or "hard" to understand. We explained that most of the sentences should be considered easy to understand, but that a few would be hard to understand. We further explained that the purpose of the exercise was to vet our stimuli for future experiments. Upon exit, participants were asked whether they suspected that the pen manipulation was designed to influence emotion, and we removed the data for those few (4) who responded in the affirmative.

\section{Results and Discussion}

On average, 94\% of the sentences were judged as "easy to understand." Analyses of the judgment times are only for sentences that were judged "easy," and we omitted trials with judgment times greater than $2.5 S D \mathrm{~s}$ from each participant's condition mean judgment time. The data (Table 2) replicated those of Experiment 1. For the judgment times, the interaction between the pen condition and sentence valence was significant $[F(1,37)=$ $\left.7.52, M S_{\mathrm{e}}=97,781, p=.01\right] .{ }^{3}$ However, the interaction was also significant for judgment accuracy $[F(1,37)=$ $\left.8.39, M S_{\mathrm{e}}=.001, p=.01\right]$, suggesting the possibility of a speed-accuracy trade-off. ${ }^{4}$ We reasoned that participants might have needed some experience with the sentences before they could establish a consistent criterion for determining whether a particular sentence was easy or hard to understand and that variability in this criterion may have affected the accuracy (and speed) of judgments in the first part of the experiment. Thus, we analyzed the data from the first half and the second half of the experiment separately. Consistent with our reasoning, for the first half of the experiment the interaction was significant for judgment accuracy $\left[F(1,37)=7.14, M S_{\mathrm{e}}=.002, p=.01\right]$ but was not significant for the judgment times $(p>.3)$. For the second half of the experiment, the interaction was significant for judgment times $\left[F(1,37)=5.92, M S_{\mathrm{e}}=116,912, p=\right.$ $.02]$ but not for judgment accuracy $(p>3)$. Consequently,

Table 2

Judgment Times (RTs, in Milliseconds) and Proportions of "Easy" Judgments (Judg.) From Experiment 2

\begin{tabular}{|c|c|c|c|c|}
\hline \multirow[b]{3}{*}{ Pen Condition } & \multicolumn{4}{|c|}{ Sentence Valence } \\
\hline & \multicolumn{2}{|c|}{ Pleasant } & \multicolumn{2}{|c|}{ Unpleasant } \\
\hline & RT & Judg. & RT & Judg. \\
\hline \multicolumn{5}{|c|}{ First Half of the Experiment } \\
\hline Teeth (smiling) & 3,442 & .93 & 3,489 & .95 \\
\hline Lips (frowning) & 3,429 & .94 & 3,353 & .93 \\
\hline \multicolumn{5}{|c|}{ Second Half of the Experiment } \\
\hline Teeth (smiling) & 3,122 & .95 & 3,235 & .95 \\
\hline Lips (frowning) & 3,268 & .95 & 3,112 & .94 \\
\hline
\end{tabular}

it is unlikely that the interaction in the judgment time data is due solely to a speed-accuracy trade-off.

\section{EXPERIMENTS 3A AND 3B}

Experiments 1 and 2 show that covert manipulation of emotional facial posture (i.e., participants are unaware of the emotion manipulation) interacts with sentence valence in comprehension. Thus, the data are consistent with a simulation account in which neural systems that function during emotional processing also function during language comprehension. Several questions remain, however. For example, is the emotion simulation functional? That is, does it have a causal role in the comprehension of language about emotional events? The data are consistent with such a claim, but do not compel it. Another question concerns the linguistic level at which the simulation is evoked. For instance, simulation may be evoked at the lexical level, as was suggested by Hauk et al. (2004). Lexical-level models of language comprehension can be made consistent with a simulation account by supposing that words automatically activate other associated concepts, including emotional concepts. As a good example, affect priming theory (Bower, 1981, 1991) can be applied in that the pen manipulation activates an emotion node (e.g., the "happy" node), which in turn spreads activation to words that are associated with that emotion (e.g., lover and embrace in the sentence You and your lover embrace after a long separation). On balance, words that occur in pleasant sentences receive more positive emotional activation and/or less negative emotional activation than words occurring in unpleasant sentences. Thus, the faster judgment times that were observed in Experiments 1 and 2 could have resulted from the faster processing of individual words that were automatically primed by the pen-activated emotion node.

In Experiment 3A and 3B, we used the pen manipulation in a lexical decision task to test the lexical account of Experiments 1 and 2. We constructed prime-target pairs with two types of prime words (neutral or associated) and two types of targets (word or nonword). In Experiment 3A, target words were taken from the sentences used in Experiments 1 and 2. In Experiment 3B, target words were strongly valenced positive and negative words that were taken from the ANEW database (Bradley \& Lang, 1999). Lexical decisions should be faster for word targets preceded by associated primes than when preceded by neutral primes. In addition, if the pen activates semantic associations, it should prime emotion-congruent targets, resulting in faster lexical decisions in both the associated word prime and neutral word prime conditions.

\section{Method}

In Experiment 3A, 38 participants from the Madison, Wisconsin area were recruited and were paid $\$ 5$ for their participation. The independent variables were prime type (neutral or associated), target type (word or nonword), pen condition (pen-in-teeth or pen-inlips), and source-sentence valence (positive or negative). To derive the 96 target words, 24 different participants were asked to indicate the single word "most central to the meaning of the sentence" for sentences used in Experiments 1 and 2. The target word was 
the word chosen most often by these participants. On average, the target words were chosen by $51 \%$ of participants (see Table 3 for examples). Pronounceable nonword targets were constructed by transposing the letters of the target words. Semantically associated primes were generated for each target word using the Edinburgh associative thesaurus database. ${ }^{5}$ The two types of primes were crossed with the two types of targets, creating 192 pairs. These pairs were repeated so that each one occurred once for each participant in the pen-in-teeth and pen-in-lips conditions, resulting in 384 trials. The 384 trials were divided into 8 blocks of 48 , and each block contained 12 exemplars of each pair combination. Participants were told that the purpose of the pen manipulation was to investigate how suppression of the speech articulators (the teeth and the lips) would affect word recognition. Participants switched pen conditions after each block of 48 trials.

\section{Results}

Mean accuracy was $95 \%$ correct, with a range of $85 \%-99 \%$ correct. Analyses were conducted on lexical decision times for the word targets for correct trials only, and all times greater than 2.5 SDs from each participant's mean were eliminated. The relevant means are presented in Table 4 . There were no statistically reliable effects due to the pen manipulation [for pen condition, $F(1,37)=$ $1.06, M S_{\mathrm{e}}=5,826, p=.309$, and for the interaction of pen condition and source-sentence valence (see the pen priming effect in Table 4), $F(1,37)=0.73, M S_{\mathrm{e}}=2,104$, $p=.399] .{ }^{6}$ The absence of any effect that was due to the pen manipulation is not likely to reflect low statistical power, because the experiment was powerful enough to produce a strong effect of word prime condition (see the word priming effect in Table 4) $\left[F(1,37)=17.12, M S_{\mathrm{e}}=\right.$ $87,925, p<.001] .{ }^{7}$ Thus, the experiment demonstrates substantial word-to-word priming, but little or no pen-toword priming.

In Experiment 3B, an additional 59 participants werein an otherwise identical task and procedure-given strongly valenced positive and negative target words taken from the ANEW database (Bradley \& Lang, 1999). Again, there were no effects that were due to the pen manipulation ( $F<1$ for all pen condition $\times$ word valence interactions), but there was a significant word priming effect with faster identification times for targets preceded by an associated prime than by a neutral prime $\left[F(1,58)=35.89, M S_{\mathrm{e}}=\right.$ $218,034, p<.001] .{ }^{8}$ In conclusion, the pen manipulation that influenced reading judgments and comprehension in Experiments 1 and 2 primes neither the same words in isolation (Experiment 3A) nor strongly valenced words
Table 3

Stimulus Examples From Experiment 3

\begin{tabular}{llll}
\hline & Word & Nonword & Associate \\
\hline Pleasant & embrace & bermace & kiss \\
& gorgeous & rogogsue & handsome \\
& newborn & bonrewn & infant \\
& partner & raptern & companion \\
Unpleasant & police & clipoe & arrest \\
& exam & maxe & grade \\
& fin & nif & shark \\
& insult & sulint & offend \\
\hline
\end{tabular}

(Experiment 3B). We concluded that the effects revealed in Experiments 1 and 2 are unlikely to arise from emotion simulation activated at initial lexical access and that lexical level accounts - such as those of Bower (1981, 1991) — need to be modified to account for these data. For example, an elaborated version of APT suggests that mood congruence results from lexical-level associations that are uncovered by extensive processing (Forgas, 1995). Alternatively, emotion simulation may affect language beyond the lexical level.

\section{GENERAL DISCUSSION}

In Experiments 1 and 2, the manipulation of facial posture influenced the speed with which participants responded to emotionally valenced sentences. In contrast, in Experiments 3A and 3B, the same manipulation failed to influence the speed of responding in a lexical decision task, even for strongly valenced target words. This pattern of results suggests that emotion simulation during language comprehension is not a lexical-level phenomenon, but instead affects comprehension at the level of the phrase or sentence. This finding contributes to the following general conclusion: Different neural and bodily systems can affect simulation at different levels of language processing. Thus, action systems appear to affect processing at both the lexical (see, e.g., Hauk et al., 2004) and sentential (e.g., Glenberg \& Kaschak, 2002) levels, whereas simulation using emotional systems is predominantly a sentence- or phrase-level phenomenon. An exception to this conclusion is that lexical processing on words that directly name emotions (e.g., happy) can be affected by emotional state (Niedenthal, Halberstadt, \& Setterlund, 1997). This overall pattern might be explained in terms of

Table 4

Lexical Decision Times (LDTs, in Milliseconds) and Proportions Correct (Prop.) for Word Targets in Experiment 3

\begin{tabular}{|c|c|c|c|c|c|c|c|}
\hline \multirow[b]{2}{*}{ Source Sentence } & \multirow[b]{2}{*}{ Pen Condition } & \multicolumn{2}{|c|}{ Neutral Prime } & \multicolumn{2}{|c|}{ Associated Prime } & \multirow[b]{2}{*}{ Word Priming Effect ${ }^{\mathrm{a}}$} & \multirow[b]{2}{*}{$M^{\mathrm{b}}$} \\
\hline & & LDT & Prop. & LDT & Prop. & & \\
\hline \multirow[t]{3}{*}{ Pleasant } & Teeth (smiling) & 631 & .96 & 609 & .98 & 22 & 620 \\
\hline & Lips (frowning) & 637 & .96 & 609 & .96 & 28 & 623 \\
\hline & Pen priming effect ${ }^{c}$ & & & & & & -3 \\
\hline \multirow[t]{3}{*}{ Unpleasant } & Teeth (smiling) & 631 & .95 & 600 & .97 & 31 & 616 \\
\hline & Lips (frowning) & 641 & .96 & 602 & .98 & 39 & 622 \\
\hline & Pen priming effectc & & & & & & -6 \\
\hline
\end{tabular}

aDifference between neutral and associated prime. bAverage of neutral and associated prime. cDifference between mean for lips and mean for teeth. 
frequency and consistency of pairing of linguistic expressions with experiences. Action words-such as lick and kick, which were used by Hauk et al. (2004) — may be consistently paired with the literal actions (at least during acquisition). Thus, whenever these words are perceived, they elicit activation in the motor system. Similarly, words that directly name emotions (e.g., happy) may be consistently paired with mood. In contrast, the emotional content that is suggested by a word such as embrace (Experiment 3) may be less consistent and depend on the sentential context (e.g., contrast embrace your lover and embrace of the snake). In this case, detectable emotion simulation is only apparent at the sentential or phrasal level.

Why should being in (or the ease of entering) a particular emotional state facilitate the comprehension of the sentence? We outline an account based on current theories of emotion in conjunction with the indexical hypothesis (IH; Glenberg \& Robertson, 1999). According to the IH, language comprehension requires three overlapping processes. The first is mapping or indexing words and phrases to objects in the environment or analogical perceptual symbols (Barsalou, 1999). The second process is the derivation of affordances (Gibson, 1979) - that is, how one can interact with the objects. Affordances depend on characteristics of the body, such as size and morphology. For example, a chair affords sitting for a human, but not for a whale, and a chair affords hiding for a child, but not for an adult. Third, syntax guides the combination of affordances to accomplish goals (Chambers, Tanenhaus, \& Magnuson, 2004; Kaschak \& Glenberg, 2000). In brief, the IH proposes that language comprehension results from a simulation of the actions that underlie the meaning of the sentence.

Several contemporary theories of emotion (see, e.g., Barrett, 2006; Frijda, 1986) propose that emotion prepares the body for appropriate actions. For example, positive affect prepares the body to approach, whereas negative affect prepares the body to avoid. Because emotions produce physical changes in the body (Davidson, 1994; Levenson, 2003; McIntosh, Zajonc, Vig, \& Emerick, 1997), emotions literally change affordances. For example, changes in blood pressure, heart rate, and breathing that are associated with excitement literally change the body so that the situation affords actions - such as the rapid bounding up a steep staircase to greet a loved one - that would not otherwise be possible.

Within this framework, consider two ways in which emotion might influence language. First, bodily actions of approach and withdrawal underlie the meanings of many pleasant and unpleasant words (e.g., embrace). Thus, neural areas that control such actions may also be involved in the words' representation, much as in Hauk et al. (2004). Accordingly, Chen and Bargh (1999) have shown that positive valenced words (e.g., love) are identified faster when the response involves an approach action (arm flexion) rather than a withdrawal action (arm extension) and vice versa for negative valenced words (e.g., hate). The discrepancy between these findings and those of Experiment 3 may be due to motor variables that are associated with the pen manipulation (e.g., planning, fatigue) and warrants further study.
Second, consider how congruent emotions could facilitate understanding a sentence such as As you step into the room, your friends shout, "Surprise!" When experiencing positive affect, the body is prepared to approach. According to the $\mathrm{IH}$, sentences are understood by simulating actions underlying the sentence. Much as positive affect facilitates the actions underlying literal approach, we propose that positive affect enhances the simulation of approach actions. Thus, affective state will modulate simulation requiring approach, and the interactions observed in Experiments 1 and 2 would be observed. Because emotion affects action simulation beyond the lexical level, it has no detectable role in the lexical decision task of Experiments 3A and 3B.

\section{AUTHOR NOTE}

This work was supported by NSF Grants BCS-0315434 and INT0233175 to A.M.G. and a grant from the German Academic Exchange Service (DAAD) to M.R. Any opinions, findings, and conclusions or recommendations expressed in this material are those of the authors and do not necessarily reflect the views of the NSF. We thank Brianna Buntje, Sheila Simhan, Terina Yip, and Bryan Webster for data collection and discussion of these results. Address correspondence to D. A. Havas, 1202 W. Johnson St., Department of Psychology, University of Wisconsin, Madison, WI 53706 (e-mail: dahavas@wisc.edu).

Note-Accepted by David A. Balota's editorial team.

\section{REFERENCES}

Barrett, L. F. (2006). Are emotions natural kinds? Perspectives on Psychological Science, 1, 28-58.

Barsalou, L. W. (1999). Perceptual symbol systems. Behavioral \& Brain Sciences, 22, 577-660.

Bower, G. H. (1981). Mood and memory. American Psychologist, 36, 129-148.

Bower, G. H. (1991). Mood congruity of social judgments. In J. P. Forgas (Ed.), Emotion and social judgments (pp. 31-53). Oxford: Pergamon.

Bradley, M. M., \& Lang, P. J. (1999). Affective norms for English words (ANEW). Gainesville, FL: University of Florida, NIMH Center for the Study of Emotion and Attention.

Buccino, G., Riggio, L., Melli, G., Binkofski, F., Gallese, V., \& Rizzolatti, G. (2005). Listening to action-related sentences modulates the activity of the motor system: A combined TMS and behavioral study. Cognitive Brain Research, 24, 355-363.

Carr, L., Iacoboni, M., Dubeau, M., Mazziotta, J. C., \& Lenzi, G. L. (2003). Neural mechanisms of empathy in humans: A relay from neural systems for imitation to limbic areas. Proceedings of the $\mathrm{Na}$ tional Academy of Sciences, 100, 5497-5502.

Chambers, C. G., Tanenhaus, M. K., \& Magnuson, J. S. (2004). Actions and affordances in syntactic ambiguity resolution. Journal of Experimental Psychology: Learning, Memory, and Cognition, 30, 687-696.

Chen, M., \& BARgh, J. (1999). Consequences of automatic evaluation: Immediate behavioral predispositions to approach or avoid the stimulus. Personality \& Social Psychology Bulletin, 25, 215-224.

DAVIDSON, R. J. (1994). Complexities in the search for emotion-specific physiology. In P. Ekman \& R. J. Davidson (Eds.), The nature of emotion: Fundamental questions (pp. 237-242). New York: Oxford University Press.

Decety, J., \& Grèzes, J. (2006). The power of simulation: Imagining one's own and other's behavior. Brain Research, 1079, 4-14.

DE GELDER, B. (2006). Towards the neurobiology of emotional body language. Nature Reviews Neuroscience, 7, 242-249.

DimBERG, U. (1982). Facial reactions to facial expressions. Psychophysiology, 19, 643-647.

Dimberg, U., Thunberg, M., \& Elmehed, K. (2000). Unconscious facial reactions to emotional facial expressions. Psychological Science, 11, 86-89. 
Forgas, J. P. (1995). Mood and judgment: The affect infusion model (AIM). Psychological Bulletin, 117, 39-66.

FRIJDA, N. H. (1986). The emotions. Cambridge: Cambridge University Press.

Gibson, J. J. (1979). The ecological approach to visual perception. Boston: Houghton Mifflin.

Glenberg, A. M., \& KaschaK, M. P. (2002). Grounding language in action. Psychonomic Bulletin \& Review, 9, 558-565.

Glenberg, A. M., \& Robertson, D. A. (1999). Indexical understanding of instructions. Discourse Processes, 28, 1-26.

Hauk, O., Johnsrude, I., \& Pulvermüller, F. (2004). Somatotopic representation of action words in human motor and premotor cortex. Neuron, 41, 301-307.

InNes-Ker, Å. [H.], \& Niedenthal, P. M. (2002). Emotion concepts and emotional states in social judgment and categorization. Journal of Personality \& Social Psychology, 83, 804-816.

Johnson, E. J., \& TVERSKy, A. (1983). Affect, generalization, and the perception of risk. Journal of Personality \& Social Psychology, 45, 20-31.

KaschaK, M. P., \& Glenberg, A. M. (2000). Constructing meaning: The role of affordances and grammatical constructions in sentence comprehension. Journal of Memory \& Language, 43, 508-529.

Kaschak, M. P., Madden, C. J., Therriault, D. J., Yaxley, R. H., Aveyard, M., Blanchard, A., \& ZWaAn, R. A. (2005). Perception of motion affects language processing. Cognition, 94, B79-B89.

Larsen, R. J., Mercer, K. A., \& Balota, D. A. (2006). Lexical characteristics of words used in emotional Stroop experiments. Emotion, 6, 62-72.

LeVenson, R. W. (2003). Blood, sweat, and fears: The autonomic architecture of emotion. Annals of the New York Academy of Sciences, 1000, 348-366.

Levenson, R. W., Ekman, P., \& Freisen, W. V. (1990). Voluntary facial action generates emotion-specific autonomic nervous system activity Psychophysiology, 27, 363-384.

McIntosh, D. N., ZaJonc, R. B., Vig, P. S., \& Emerick, S. W. (1997). Facial movement, breathing, temperature, and affect: Implications of the vascular theory of emotional efference. Cognition \& Emotion, 11, 171-195.

Niedenthal, P. M., Brauer, M., Halberstadt, J. B., \& Innes-Ker, A. H. (2001). When did her smile drop? Facial mimicry and the influences of emotional state on the detection of change in emotional expression. Cognition \& Emotion, 15, 853-864.

Niedenthal, P. M., Halberstadt, J. B., \& Setterlund, M. B. (1997). Being happy and seeing "happy": Emotional state mediates visual word recognition. Cognition \& Emotion, 11, 403-432.

OATLEY, K. (1994). A taxonomy of the emotions of literary response and a theory of identification in fictional narrative. Poetics, 23, 53-74.

Raaijmakers, J. G. W., Schrijnemakers, J. M. C., \& Gremmen, F. (1999). How to deal with "The language-as-fixed-effect fallacy": Common misconceptions and alternative solutions. Journal of Memory \& Language, 41, 416-426.

Rizzolatti, G., Fogassi, L., \& Gallese, V. (2001). Neurophysiologi- cal mechanisms underlying the understanding and imitation of action. Nature Reviews Neuroscience, 2, 661-670.

Strack, F., Martin, L. L., \& StepPer, S. (1988). Inhibiting and facilitating conditions of the human smile: A nonobtrusive test of the facial feedback hypothesis. Journal of Personality \& Social Psychology, 54, 768-777.

Tettamanti, M., Buccino, G., Saccuman, M. C., Gallese, V., DanNA, M., ScIFo, P., ET AL. (2005). Listening to action-related sentences activates fronto-parietal motor circuits. Journal of Cognitive Neuroscience, 17, 271-281.

Velten, E. (1968). A laboratory task for induction of mood states. Behaviour Research \& Therapy, 6, 473-482.

ZWAAN, R. A., \& TAYLOR, L. J. (2006). Seeing, acting, understanding: Motor resonance in language comprehension. Journal of Experimental Psychology: General, 135, 1-11.

\section{NOTES}

1. We are very grateful to Ira Fischler who provided us with the stimulus sentences.

2. Although Raaijmakers, Schrijnemakers, and Gremmen (1999) suggested that item analyses are inappropriate for designs such as this one, we report them at the request of reviewers. Both the subject analysis and the item analysis were performed after eliminating one pleasant and one unpleasant sentence with unusually high error rates $(22 \%$ and $33 \%$, respectively). The interaction between pen condition and sentence valence $\left[F(1,92)=3.24, M S_{\mathrm{e}}=50,425\right]$ was just shy of being statistically significant $(p=.07)$.

3. An item analysis similar to that reported in note 2 was conducted. Both the subject and item analyses excluded one unpleasant and one pleasant item with an unusually high error rate $(50 \%)$ and an inadequate cell count that was due to trimming, respectively. The interaction between pen condition and sentence valence was again nearly significant for raw reading times $\left[F(1,92)=3.41, M S_{\mathrm{e}}=224,351, p=.06\right]$.

4. The term judgment accuracy reflects participants' accuracy in confirming our intuition - and those of norming subjects - that all sentences were comprehensible.

5. Available at www.eat.rl.ac.uk.

6. There was no statistically significant correlation between the size of the pen effect and the number of participants who selected the target word from the sentence.

7. The results of an item analysis were consistent with the results from the subject analysis: a large effect of word priming $[F(1,94)=33.27$, $\left.M S_{\mathrm{e}}=1,050\right]$ and no effect for the pen manipulation or its interaction with source-sentence valence $(F \mathrm{~s}<1.89)$.

8 . The results of an item analysis were consistent with the results from the subject analysis: a large effect of word priming $[F(1,94)=76.23$, $\left.M S_{\mathrm{e}}=1,355\right]$ and no effect for the pen manipulation or its interaction with source-sentence valence $(F \mathrm{~s}<1.48)$.

(Manuscript received December 9, 2005; revision accepted for publication July 28, 2006.) 